\title{
Small-world effects in a modified epidemiological model with mutation and permanent immune mechanism
}

\author{
Shengli Cao - Peihua Feng - Wei Wang • \\ Yayun Shi · Jiazhong Zhang $\mathbb{D}$
}

Received: 29 December 2020 / Accepted: 4 May 2021 / Published online: 11 May 2021

(C) The Author(s), under exclusive licence to Springer Nature B.V. 2021

\begin{abstract}
Pandemic with mutation and permanent immune spreading in a small-world network described is studied by a modified SIR model, with consideration of mutation-immune mechanism. First, a novel mutation-immune model is proposed to modify the classical SIR model to simulate the transmission of mutable viruses that can be permanently immunized in small-world networks. Then, the influences of the size, coordination number and disorder parameter of the small-world network on the spread of the epidemic are analyzed in detail. Finally, the influences of mutation cycle and infection rate on epidemic transmission in small-world network are investigated further. The results show that the structure of the small-world network and the virus mutation cycle have an important impact on the spread of the epidemic. For viruses
\end{abstract}

S. Cao $\cdot$ W. Wang $\cdot$ J. Zhang $(\varangle)$

School of Energy and Power Engineering, Xi' an Jiaotong University, Xi' an 710049, China

e-mail: jzzhang@mail.xjtu.edu.cn

W. Wang

e-mail: weiwwang@qq.com

S. Cao

e-mail: cs11993@stu.xjtu.edu.cn

P. Feng · Y. Shi

State Key Laboratory for Strength and Vibration of

Mechanical Structures, School of Aerospace Engineering,

Xi'an Jiaotong University, Xi' an 710049, People's

Republic of China

e-mail: fphd2017@xjtu.edu.cn

Y. Shi

e-mail: yayunshi@xjtu.edu.cn that can be permanently immunized, virus mutation is equivalent to making the immune cycle of human beings from infinite to finite. The dynamical behavior of the modified SIR epidemic model changes from an irregular, low-amplitude evolution at small disorder parameter to a spontaneous state of wide amplitude oscillations at large disorder parameter. Moreover, similar transition can also be found in increasing mutation cycle parameter. The maximum valid variation mutation decreases with the increase of disorder parameter and coordination number, but increase with respect to system size. In addition above, as the infection rate increases, the fraction of the infected increases and then decreases. As the mutation cycle increases, the time-average fraction of the infected and the infection rate corresponding to the maximum time-average fraction of the infected also decrease. As one conclusion, the results could give a deep understanding Pandemic with mutation and permanent immune spreading, from viewpoint of small-world network.

Keywords Pandemic · Small-world network · SIR model $\cdot$ Mutation

\section{Introduction}

In December 2019, a new coronavirus, now named SARS-CoV-2, resulted in a series of acute atypical respiratory diseases in Wuhan, Hubei Province, China. The disease caused by this virus is named as COVID- 
19. This virus with high transmission and mortality spread from human to human, and quickly triggered a global pandemic [1]. More, COVID-19 has gave rise to great harm to the health of patients and even leads to death. As of 18 April 2021, more than 141.4 million cases have been confirmed, with over 3 million deaths attributed to COVID-19. Starting from the end of July 2020, a potential second wave of infections seemed to appear with different severity in different areas [2,3]. Indeed, after a decrease during summer, the number of patients with the disease increased again, suggesting the origin of the second wave outbreaks of infectious diseases. At present, the epidemic still plagues the society, making continuous loss of human life and economy [4].

Early in the pandemic, SARS-CoV-2 virus acquired a D614G mutation in the spike protein. With the rapid increase of mutation frequency, it has become the dominant form of the virus globally [5]. Although this mutation does not appear to increase disease severity, it gives the virus a fitness advantage for transmission [6]. From August to September in 2020, a variant of SARS CoV2 related to mink infection was found in the North Jutland peninsula of Denmark, which was then transmitted to humans, but did not give rise to widespread transmission [7]. A novel SARS-CoV-2 variant, VOC 202012/01, emerged in southeast England in November 2020. VOC 202012/01 is defined by 17 mutations, among which eight are located in the spike protein. Previous studies have shown that VOC 202012/01 is 56\% more transmissible (95\% credible interval across three regions $50-74 \%$ ) than preexisting variants of SARSCoV-2 [8]. Obviously, virus mutation has an important impact on the spread of the epidemic. The immune response recognizes foreign molecules in a highly specific way. Immune cells or antibodies that recognize proteins from one pathogen strain may not recognize similar proteins from another pathogen strain. Therefore, mutations in the virus may increase the infection rate of the virus, and may even render the vaccine useless. Thus, the transmission characteristics of mutated viruses in the population is a subject worthy of study.

To understand the spread of disease and identify possible outbreak control methods, it is necessary to study the impact of population structure and contact network on virus transmission. Each individual in the population is regarded as a node, and the interaction between nodes can be described by complex network. Pathogens attach to nodes and spread in the network through the interaction of nodes. In recent years, the mathematical modeling of network dynamics process has produced a large number of results, which help to understand the complexity of epidemic waves and predict the consequences and risks of spreading diseases [9-11]. One of the important attributes of the contact network on virus transmission is the small-world property, which appears in many socio-spatial real-world networks [12]. Small-world property refers to the fact that individual nodes are frequently connected in its local space, but they are also connected to distant nodes through a small number of intermediate nodes. Watts and Strogatz proposed the first small-world network model, which exists in the network spectrum between completely random and completely regular network models, and has the high clustering of regular network and the high connectivity of random network [13]. Numerous studies have shown that small-world network is a ubiquitous infrastructure, which exists in all kinds of real-world networks, including social [14], information [15], technology and biological networks [16].

Small-world network is especially suitable for describing the spatial relationship between hosts in the process of virus transmission [17]. Small et al. [18] used the small-world network model to analyze SARS and proved that this structure can reliably generate quantitative simulations similar to real data. The small world network model not only captures the obvious random fluctuations in the SARS reporting data in Hong Kong, but also reproduces small outbreaks due to the so-called super-spreaders. Wang et al. [19] studied the synchronization problem of continuous time dynamical system networks with small-world connections. Li et al. [20] introduced a nonlinear SIQS epidemic model based on small-world network. By using complex network theory and Lyapunov function, the basic reproduction number and global stability of disease-free equilibrium and endemic equilibrium are obtained. In addition to these studies, there are also a large number of studies using small-world network to study the epidemic [21-25].

As the structure of networks always affects dynamics occurring on the networks, the interplays between dynamics occurring on complex networks and the structural properties of complex networks have been considered. Epidemic infection is one of the mainstream studies on complex networks [16], and many studies have focused on the impact of small-world net- 
work structure on epidemic transmission [26-29]. Most impressively, Kuperman and Abramson used the smallworld network and SIRS model to analyze the model of infectious disease transmission for different population structure [17]. Interactions within populations are described by small-world networks, from ordered lattices to random graphs. Their results show that there was a fluctuating epidemic of low infection for more ordered systems. At a finite value of the disorder of the network, a transition to self-sustained oscillations in the size of the infected subpopulation occurred. Their results fully demonstrate the impact of network structure on epidemic transmission patterns. As mentioned above, virus mutation can also have a tremendous impact on the transmission characteristics of the virus. However, this issue is not considered in Kuperman and Abramson's works.

In fact, only a small number of studies focus on the influence of virus mutation on propagation characteristics in complex networks. Because the sequence and structure of viral proteins are determined by genes, the immune response to pathogens is actually pathogen specific genes. However, in the previous studies on epidemic dynamics in the contact network, only a very small number of studies involve the virus mutation. There are mainly three virus mutation models used in the contact network to analyze the spread of the epidemic, namely, bitstring model [30,31], fitness space model [32,33] and epidemic spreading SIVR model $[34,35]$. In the bitstring model, different pathogen strains are represented by bitstrings that can mutate, where the bitstring are regarded as abstract representation of pathogen genetic codes. In this model, hosts are immune to infection by pathogens that are highly similar to pathogens with which they have been previously infected, where similarity between two strains are measured by hamming distance. This model can directly reflect the mutation of pathogens. Fitness model is to establish a functional relationship between gene variable and transmission probability. The pathogen mutates by changing the gene variable. After the mutation, the transmission probability of the new pathogen changes. Generally, the concept of the fitness landscape relates the genotype of an organism to its survival and reproductive success. In SIVRS model, virus variation factors are considered in the process of epidemic spreading based on complex networks, which can describe different contact status for different agents including the susceptible (S), the infected (I), the vari- ant $(\mathrm{V})$ and the recovered $(\mathrm{R})$ in a network. All new individuals produced can be considered as blank nodes in complex networks, which are susceptible. When a susceptible individual contacts the other infected individual, this individual may be infected with certain probability. Then an infected individual would have only three states including the infected, the variant and the recovered. The infected individuals would become the variants with certain probability affected by some factors, such as gene mutation and the indeterminacy of cells division. Similarly, an infected person may become a variant with certain probability after contacting with a variant. Usually, human body can be protected by his immune system. In particular, some infected individuals with recovery probability may become the recovered, while others will keep the infected status. Although these models are used to study the mutation in the epidemic, only Sten's [33] work applies the mutation model to the small world network model. And some of these studies do not consider individual immunity into the virus, and some studies do not consider the lifelong immunity of the virus. Therefore, further research in depth is still needed.

Actually, human beings can produce permanent immunity to some viruses, and there is no research work on the network dynamics of viruses that individuals can have permanent immunity to. To study this issue, a new model is proposed in present paper to study the transmission of viruses that can mutate and be permanently immunized. Particularly, viruses competing with the human immune system are described in this model. The human immune system will produce permanent immunity to the virus, and the virus will mutate to produce new virus to cope with the permanent immunity produced by human. Finally, this paper combines small world network and SIR model and the mutationimmune model to study the virus transmission that can mutate and be permanently immunized. Considering permanent immunity, the influences of network structure, virus infection ability and virus mutation on the dynamics of virus transmission are investigated in detail.

\section{Epidemic model}

The classical epidemic SIR model is given by the system of ordinary differential equations as following, 


$$
\begin{aligned}
\frac{\mathrm{d} S}{\mathrm{~d} t} & =-\beta \frac{S I}{N} \\
\frac{\mathrm{d} I}{\mathrm{~d} t} & =\beta \frac{S I}{N}-\gamma I \\
\frac{\mathrm{d} R}{\mathrm{~d} t} & =\gamma I
\end{aligned}
$$

In Eq. (1), a community of $N$ individuals are divided into the following three categories, namely, susceptible $(S)$, infected $(I)$ and recovered $(R)$. A susceptible individual may become infected or remain susceptible, and it can be infected by contact with infected individuals. The number of newly infected per unit time is directly proportional to the number of susceptible, and the proportional coefficient is $\beta$. Infected individuals are those individuals who have already been infected and can transmit virus to those individuals who are susceptible. An infected individual may remain infected, and can be recovered from the infected population. Similarly, the number of newly recovered per unit time is directly proportional to the number of infected, and the proportional coefficient is $\gamma$. Recovered individuals are those individuals who have recovered from the virus and are assumed to be immune. The susceptible $(S)$, infected $(I)$ and recovered $(R)$ individuals satisfy the following equations,

$N=S+I+R$

Small-world network model is considered as a potential model of social contact network, which was first discovered by Watts and Strogatz through network rewiring. It is used to describing the interaction between individuals in a population. Each network vertex of the network represents an individual. The connection between two individuals means that there is contact between them. If one of two individuals is infected and the other is susceptible, then the susceptible individual may be infected through contact with the infected individual. It is assumed that this infection occurs with a certain probability. The small-world network model is a random network based on a topological ring, and it has $N$ vertices and coordination number $2 \mathrm{~K}$, which means that each vertex is connected to $2 \mathrm{~K}$ nearest neighbors.

Network rewiring attempts to introduce randomness into a regular network, and for each vertex in the network, it adjusts each edge to another randomly selected vertex with a certain probability $p \in(0,1)$. As $p=0$, a regular lattice can be obtained. As $p \geq 0$, the progressively random graph can be obtained, and the small- world phenomenon is triggered. As $p=1$, all links are rewired, and the topology is similar to that of a completely random network. Hence, $p$ can be regarded as a disorder parameter of the network.

In order to simulate virus mutation in small world network, SIR model is modified and its dynamic process is evolved by discrete steps. In small world network, each vertex has three states, namely S, I and R state. A vertex is in $S$ state before being infected. If it is infected, it goes into an infection cycle $\tau_{I}$ and it turn into I state. The physical meaning of this period $\tau_{I}$ is the average infection period of all vertices. At the end of the infection, the infected individual reaches the recovered state. In SIR model, immunity will be produced as the infection progress of infected individuals is over.

To study the virus mutation, the virus mutation with a certain probability is considered in this paper. The new virus mutated from the existing virus will also infect individuals that are immune to the existing virus, but not yet immune to the new virus. Therefore, there may be many kinds of viruses exist in SIR model instead of one virus in traditional SIR model. If there are $k$ connected individuals infected with the same virus $v$ for an susceptible vertex, and the susceptible vertex had a probability $q$ of contagion with each infected neighbor, the probability of a susceptible being infected with this virus $v$ is as follows,

$p_{r}(v)=1-(1-q)^{k}$

Refer to the setting in Ref. [17], the value of $q$ is taken as 0.2 . If this vertex is surrounded by neighbors infected with different viruses, it is necessary to determine which virus the individual is infected with. In the following simulation, the virus with the highest probability of infection is selected from multiple virus types. If the probability of infection of multiple viruses is the same, one virus will be selected randomly. At the end of the infection cycle, the vertex enters the R state, which means permanent immunity to the virus. However, in order to study the problem of virus mutation, the R state is replaced by a special S state in the network. That is to say, a person who recovers from a certain virus will be permanently immune to that virus, but may be susceptible to other viruses. Details of this special S state are described in Sect. 3.1.

According to the above dynamic process, each vertex is characterized by a time counter $\tau_{i}(t)=$ 
$0,1,2,3 \ldots \tau_{I}$, describing its phase in the cycle of the disease. For a particular virus, the time counter of each individual will be changed according to the following rules, interval for the virus to produce a virus with new antigenic determinants in the process of mutation. In this paper, it is assumed that the time interval of the mutation oscillates near a fixed value.

$\tau_{i}(t+1)= \begin{cases}0 & \tau_{i}(t)=0 \text { and } \mathrm{i} \text {-th individual is not infected. } \\ 1 & \tau_{i}(t)=0 \text { and } \mathrm{i} \text {-th individual becomes infected } \\ \tau_{i}(t)+1 & 1 \leq \tau_{i}(t)<\tau_{I} \\ 0 & \tau_{i}(t)=\tau_{I}\end{cases}$

\section{Mutation-immune model}

\subsection{The novel mutation-immune model}

The immune response recognizes foreign molecules in a highly specific way. Individual immune cells or antibodies that recognize a protein from one pathogen strain may be unable to recognize a similar protein derived from another pathogen strain. Because protein sequence and structure are determined by the genetic content of an organism, immune responses to a pathogen are in fact specific to the pathogen's genetic content. Viruses are continuously changing as a result of genetic selection. They undergo subtle genetic changes through mutation and major genetic changes through recombination. Mutations can produce viruses with new antigenic determinants. The emergence of an antigenically new virus through mutation is called antigenic drift. Antigenically altered viruses may reinfect previously immunized hosts. However, the severity of mutations can range from zero consequence to major changes in proteins and their functions. Mutations that interfere with the basic functions of adhesion, penetration, membrane removal, replication, assembly and release are not allowed to be misused and will soon disappear from the population. However, due to the redundancy of the genetic code, many mutations are neutral, resulting in no change of virus protein, or one amino acid is replaced by amino acid with similar function. Not all mutations can persist in the virus population. Only mutations that do not destroy the basic functions can persist in a virus population. In other words, only a small amount of genetic mutation can produce antigenic drift. Therefore, although the virus is constantly mutating, it will not continuously produce viruses with new antigenic determinants. It must take a certain time
Based on the above facts, a novel mutation-immune model is proposed. Suppose that the virus mutates at every time, only one new virus will be produced. And each individual will be infected with only one virus at the same time. The virus mutation parameter $m$ is introduced. Let $m=0$ at the initial time, and add a random number (between 0 and 1) to $m$ in each time step. Then, the threshold $M_{t h}$ of virus mutation is designed to control the mutation cycle. Mutation occurs as $m$ is greater than or equal to $M_{t h}$. After mutation, the mutation parameter $m$ is reset to 0 immediately,

$m(t+1)=\left\{\begin{array}{cc}m(t)+\text { random }(0,1) & m<M_{t h} \\ 0 & m \geq M_{t h}\end{array}\right.$

Since the expectation of random number is 0.5 , the mutation parameter means that the average mutation cycle of virus is $2 m$. According to the definition of $m$, the smaller $\mathrm{m}$ is, the faster the virus mutates.

Furthermore, considering permanent immunity, the following immune mechanism are designed. An immune record array $I M$ is designed for each individual. After a infection cycle, the individual is immune to a virus. Then, the individual still returns to a special $\mathrm{S}$ state. The individual's immune array will record the type of virus recently infected and will no longer be infected by this type of virus. Based on the above, the state and immune records of each individual will be changed according to the following rules, 
$\begin{cases}\pi_{i}(t)=S, I M_{i}(t)=I M_{i}(t-1) & \tau_{i}(t)=0, \tau_{i}(t-1)=0 \\ \pi_{i}(t)=I, I M_{i}(t)=I M_{i}(t-1) & \tau_{i}(t)>0 \\ \pi_{i}(t)=S, I M_{i}(t)=\left[I M_{i}(t-1), v\right] & \tau_{i}(t)=0, \tau_{i}(t-1)=\tau_{I}(\text { Recovered from virus } v .)\end{cases}$

To simplify the complexity of the problem, it is assumed that a fixed mutation ratio of infected will be transformed into new virus infectors in each mutation. The mutation ratio is obviously a small value because only the mutation that does not destroy the basic function can persist in a virus population. Then, cases with different mutation ratios of infected $(1 \%, 2 \%, 5 \%, 10 \%$, $15 \%$ and $20 \%$ ) on small world networks, are simulated.

Actually, the value of the mutation ratio has little effect on the transmission of infection. Figure 1 shows a part of the fraction of the infected time series under different mutation ratios in the network. The 200-time steps shown are representative of the stationary state. Figure 2 shows the time-average fraction of the infected in 200-time steps after the infection curve entering the stationary state as a function of the mutated ratio. To avoid the randomness of the results, each point in Fig. 2 corresponds to a subsequent average over 100 realizations of the networks and the initial condition. From Figs. 1 and 2, it can be seen that the mutation ratio has little effect on the infection curve. Therefore, we chose $10 \%$ as the mutation rate without loss of generality.

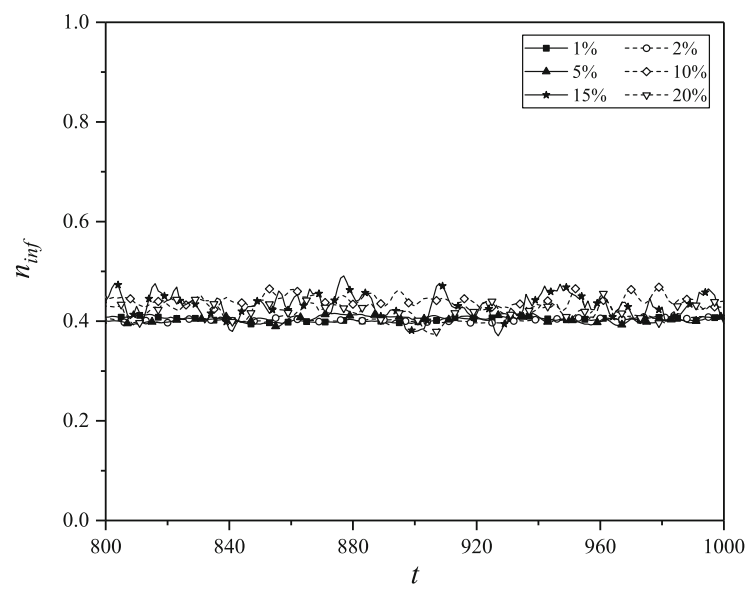

Fig. 1 Fraction of all infected as a function of time under different mutation ratio with parameters $N=10^{4}, K=3, p=$ $0.1, \tau_{I}=5, n_{\text {inf }}(0)=0.1, M_{t h}=4$

\subsection{Verification of the mutation-immune model}

To further justify the mutation-immune model, the present mutation-immune model is compared with the mutation model proposed by Girvan et al. [31]. They proposed a bitstring model in which pathogen strains are represented by bitstrings, where the bitstring is regarded as an abstract representation of a pathogen's genetic code. Hosts are immune to infection by pathogens that are highly similar to pathogens with which they have been previously infected, where similarity between two strains are measured by hamming distance $h_{t h r}$. The model consists of $N$ individuals who are either uninfected or infected with a strain represented by one of the $2^{l}$ possible bitstrings of fixed length $l$. Individuals keep a record of all the strains with which they have been infected, and these histories are defined as their memory repertoire. In every time step, each infected individual exposes $z$ others by selecting individuals uniformly at random from the entire population. The susceptibility of a contacted individual is determined by comparing the bitstring of the challenge

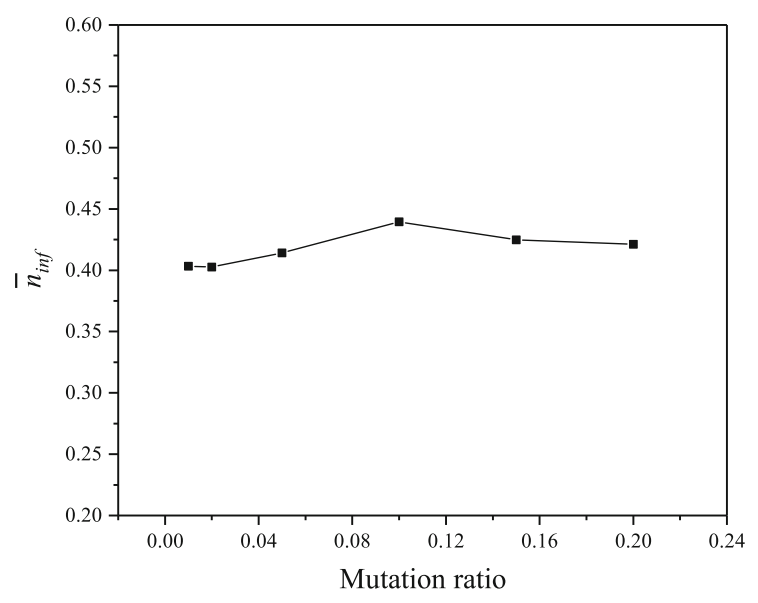

Fig. 2 Time-average fraction of the infected as a function of the mutated ratio with parameters $N=10^{4}, K=3, p=0.1, \tau_{I}=$ $5, n_{\text {inf }}(0)=0.1, M_{t h}=4$ 
Fig. 3 Boundary lines between epidemic persistence and extinction in the bitstring model and the mutation-immune model in present work

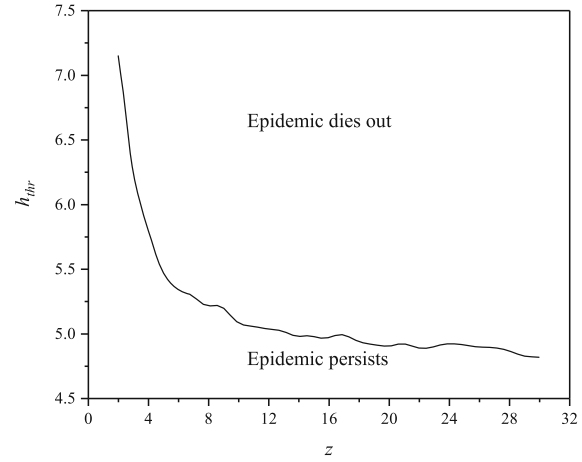

(a) Boundary between epidemic persistence and extinction in the bitstring model[31]. Results were obtained by averaging the greatest value of $h_{t h r}$ at which the pathogen persisted over 150 runs for each value of $z$.

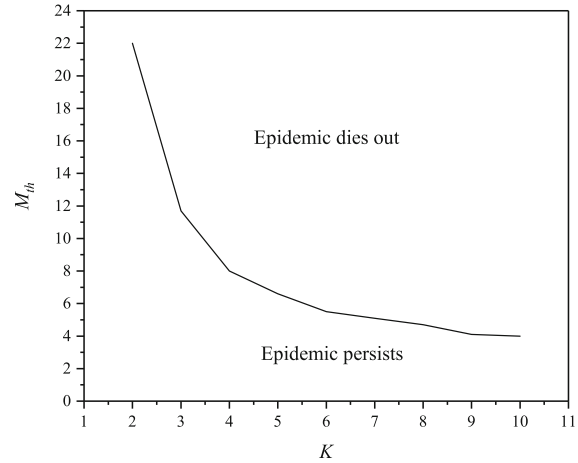

(b) Boundary between epidemic persistence and extinction in the mutation-immune model in present work with parameters $N=$ $10^{4}, \tau_{I}=5, n_{\text {inf }}(0)=0.1, M_{t h}=4$. Results are obtained by averaging the greatest value of mth at which the pathogen persisted over 150 runs for each value of $K$. strain with the bitstrings of all strains in the memory repertoire. Specifically, an individual is susceptible if $h_{\min }>h_{t h r}$, where $h_{t h r}$ is a parameter and $h_{\min }$ is the smallest hamming distance between the challenge strain and any strain in the individual's memory repertoire. With probability $m$, the challenge strain mutates by flipping one randomly chosen bit. Otherwise, the strain remains unchanged.

Obviously, the parameter $z$ in literature [31] is similar to the parameter $K$ of the small world network model in present work, and the parameter $h_{t h r}$ is equivalent to the $M_{t h}$ in this paper. It can be seen from Fig. 3 that the trend of boundary line is very similar between the mutation model in Ref. [31] and the present mutation-immune model, implying the model can be used to describe the process of virus mutation and immunity.

\section{Results and discussions}

\subsection{Influences of contact network structure on epidemic transmission}

Part of three time series is shown in Fig. 4, indicating the fraction of all infected in the system $n_{\text {inf }}$. The three curves correspond to systems with different values of the disorder parameter, $p=0.01$ (Fig. 4a), 0.2 (Fig. 4b), and 0.9 (Fig. 4c). The 400-time steps shown are representative of the stationary state. It can be clearly observed a transition from an endemic situation to an oscillatory one. As $p=0.01$, the network structure is very regular, and the fraction of infected fluctuates slightly around a fixed value. The fraction of infected is about $30 \%$, which indicates that the epidemic spreads continuously and stably in the entire network. This condition corresponds to an endemic infection with a low and persistent fraction of infected individuals. As $p$ increases to 0.1 , the fraction of infected will fluctuate strongly in a few time intervals, and the oscillation amplitude remains small for the rest of the time. As $p=0.9$, the small-world network is very similar to the random network. The amplitude of the oscillation of the infection curve becomes greater, and the infection curve has both moderate and strong oscillations. Obviously, the fluctuation amplitude of infection curve becomes larger and larger with the increase of $p$. This result is highly similar to that in Ref. [17]. In Ref. [17], the time for an individual to be immune to the virus is limited. After the immune period, the individual becomes susceptible again. It shows that the higher the randomness of the contact network, the greater the oscillation amplitude of the infected, whether in the system with permanent immunity or in the system with limited immunity cycle.

Figure 5 illustrates the fraction of infected with various mutated viruses as a function of time with different disorder parameter $p$. It is clear that competition among various viruses can be observed as $p$ is low. As $p$ increases, a certain virus will gradually become the main virus in the small-world network. As a virus is the main virus, it will make the infected population show a 
Fig. 4 Fraction of all infected as a function of time under different disorder parameters $p$ with parameters

$N=10^{4}, K=3, \tau_{I}=$ $5, n_{\text {inf }}(0)=0.1, M_{t h}=4$

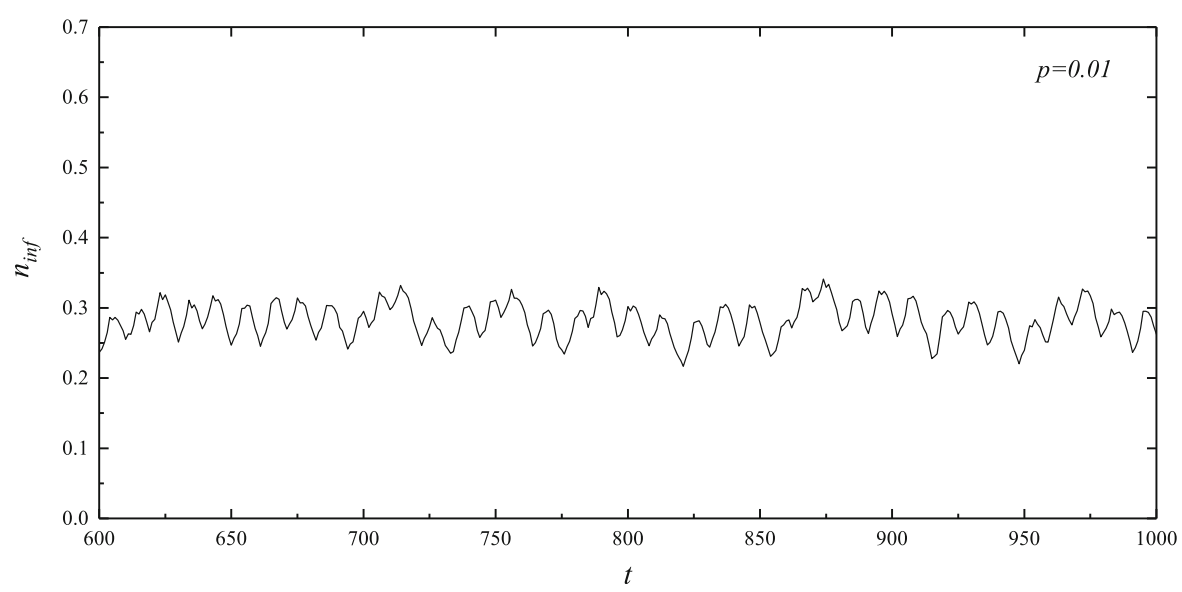

(a)

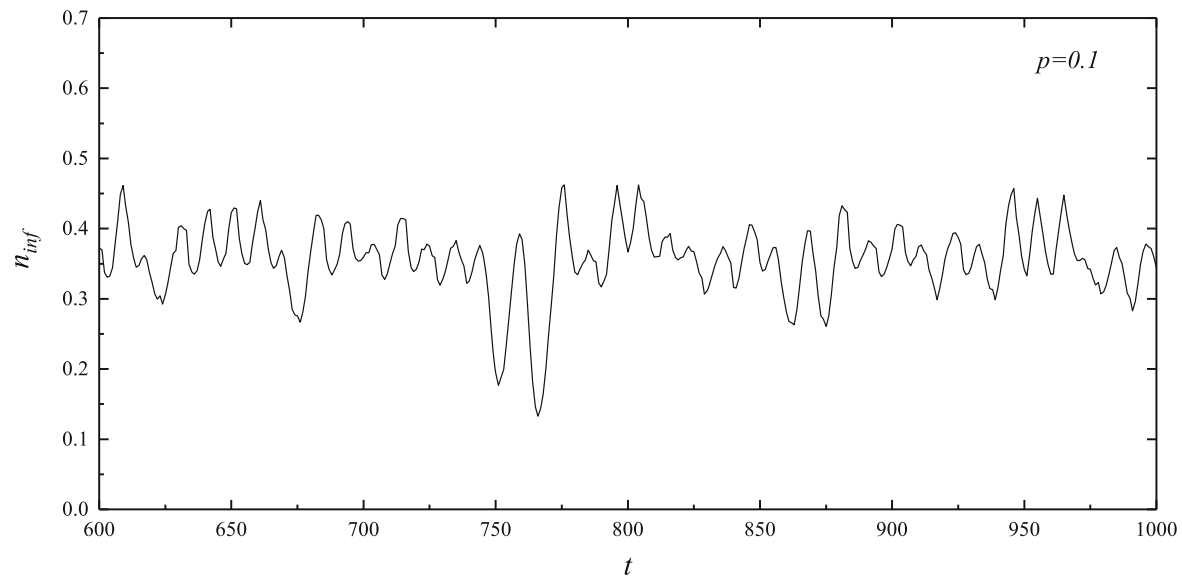

(b)

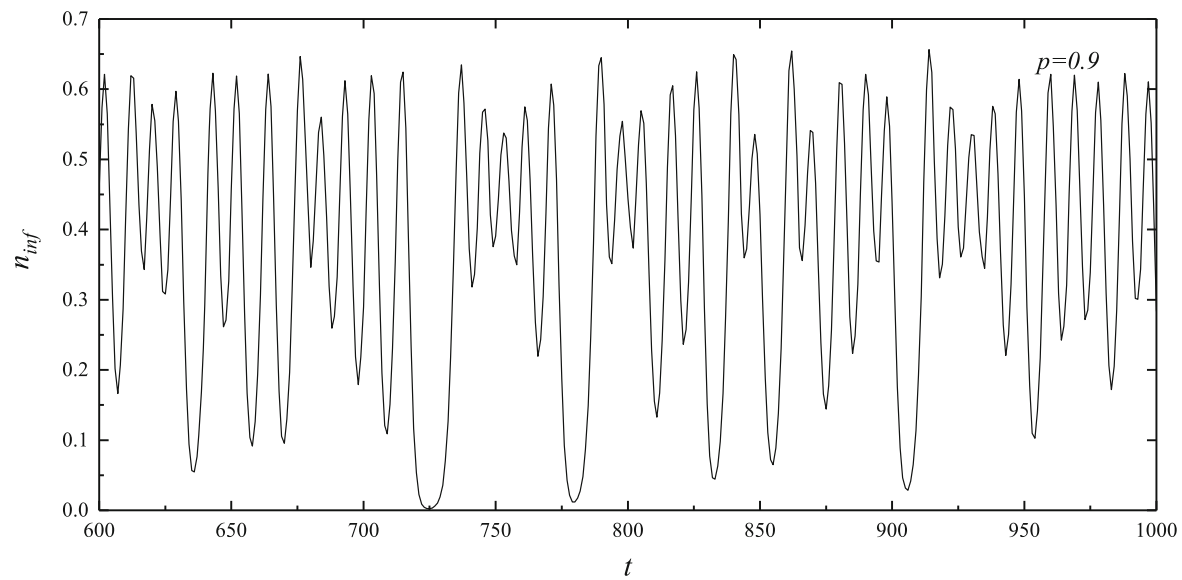

(c) 
Fig. 5 Fraction of infected with various mutated viruses as a function of time under different disorder parameters $p$ with parameters

$N=10^{4}, K=3, \tau_{I}=$

$5, n_{\text {inf }}(0)=0.1, M_{t h}=4$

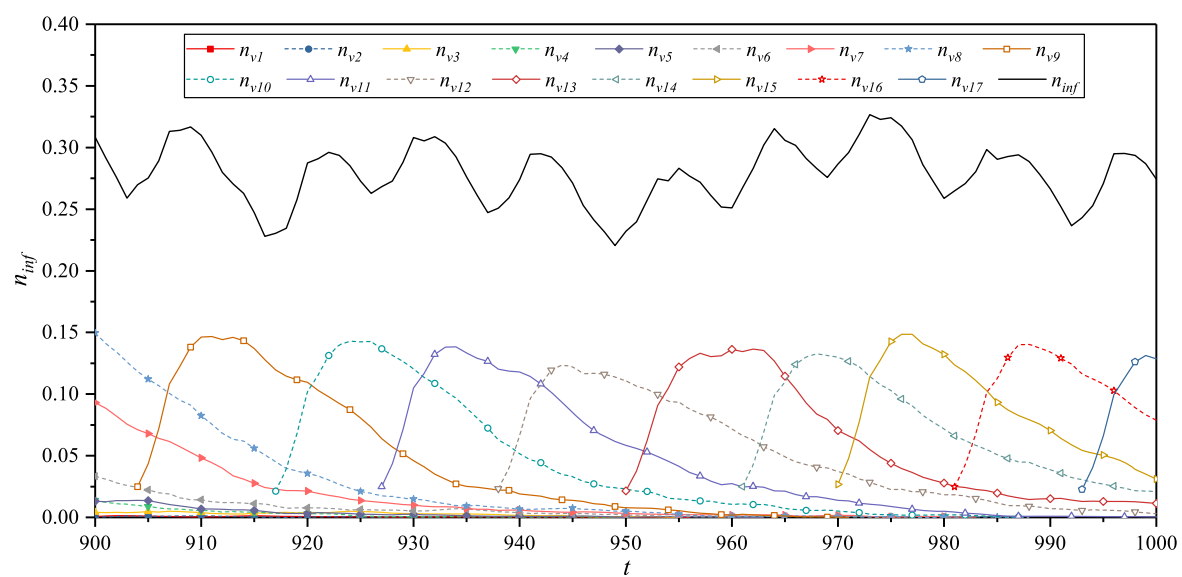

(a)

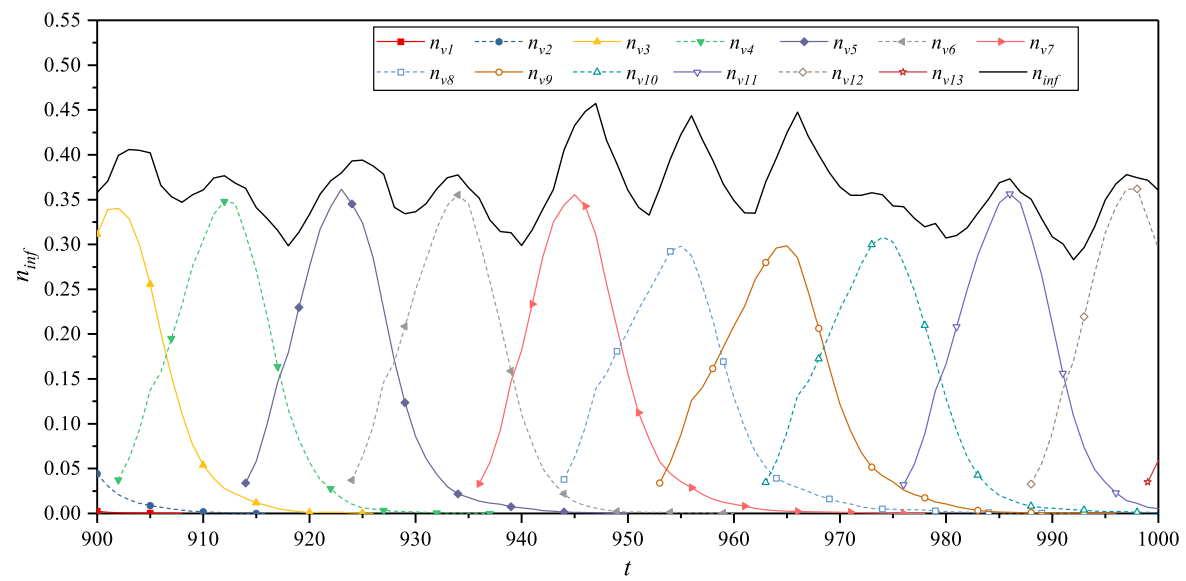

(b)

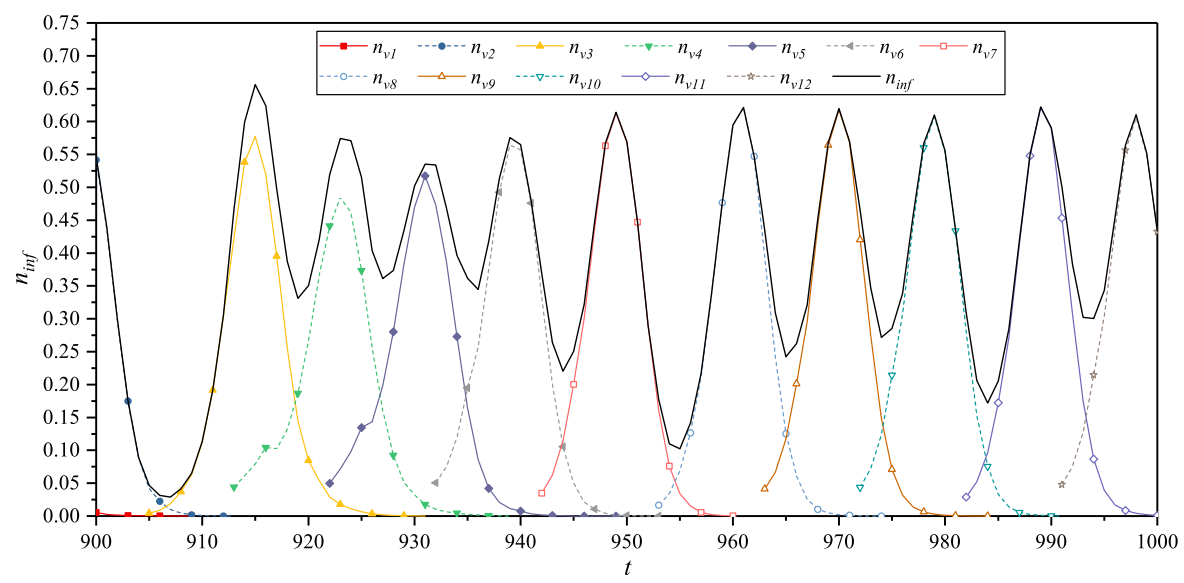

(c) 
large fluctuation trend. It shows that the structure of the network has a strong impact on the transmission characteristics of the epidemic. For each virus, one person will be permanently immune to it. With the spread of the virus, the herd immunity to the virus is produced. However, due to the mutation of the virus, the new virus will continue to spread in the population. Considering that COVID-19 is an RNA single stranded virus, which is prone to mutation, the effect of population immunity by letting people to be infected is limited. It is a better choice to develop vaccine as soon as possible, so as to realize the herd immunity.

Furthermore, the major reason for the continuous oscillation is the spontaneous synchronization of infection status of most individuals. In other words, the phases $\tau_{i}(i=1,2,3 \ldots N)$ of a large number of individuals in the epidemic cycle are synchronous. A synchronization parameter $\sigma$ is introduced to describe this behavior and is defined as [17],

$\sigma(t)=\left|\frac{1}{N} \sum_{j=1}^{N} e^{i \phi_{i}(t)}\right|$

where $\phi_{j}(t)=2 \pi\left[\tau_{j}(t)-1\right] / \tau_{I}$ is a geometrical phase corresponding to $\tau_{j}$.

Obviously, each time step has a corresponding synchronization parameter. When calculating the synchronization parameters, the case of $\tau_{j}=0(j=$ $1,2,3 \ldots N$ ) will be excluded and only the individuals in the infected state will be considered. If the system is not synchronized, the phase of each individual is widely distributed in the epidemic cycle, and the complex number $e^{i \phi_{i}(t)}$ is correspondingly distributed in the unit circle. In this case, $\sigma$ is small. By contrary, $\sigma$ is large when a large number of individuals are synchronized in the epidemic cycle.

The synchronization parameter $\sigma$ obtained as a time average of $\sigma(t)$ over 1000-time steps corresponding to system sizes $N=10^{3}, 10^{4}$ and $10^{5}$ are shown in Fig. 6 respectively. Each point corresponds to a subsequent average over several realizations of the networks and the initial condition. A transition in the synchronization can be observed as $p$ runs from 0 to 1 . The transition occurs at a value of the disorder parameter $p_{\text {critical }}=0.1$. This conclusion applicable to networks of different sizes. In other words, the size of the smallworld network has no significant effect on the transition phenomenon.

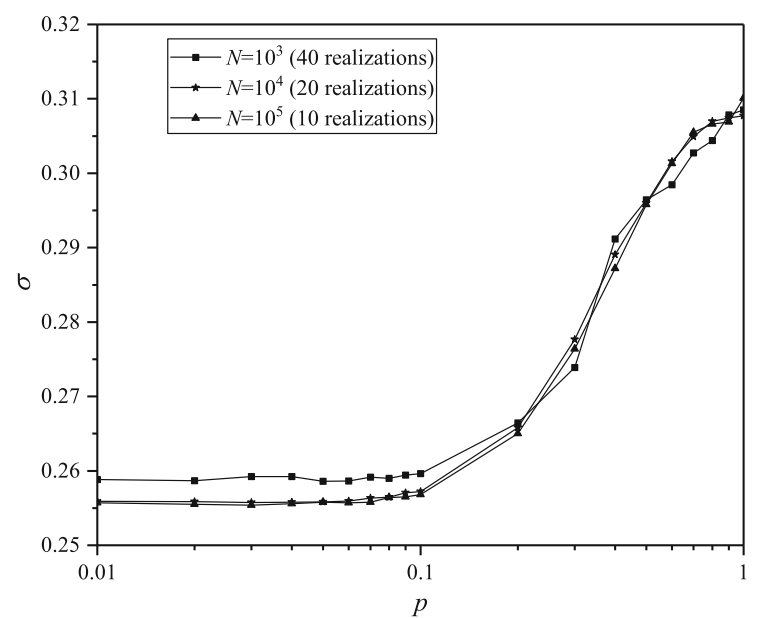

Fig. 6 Synchronization of the system as a function of the disorder parameter $p$ under different system sizes $N$ with parameters $K=3, \tau_{I}=5, n_{\text {inf }}(0)=0.1, M_{t h}=4$

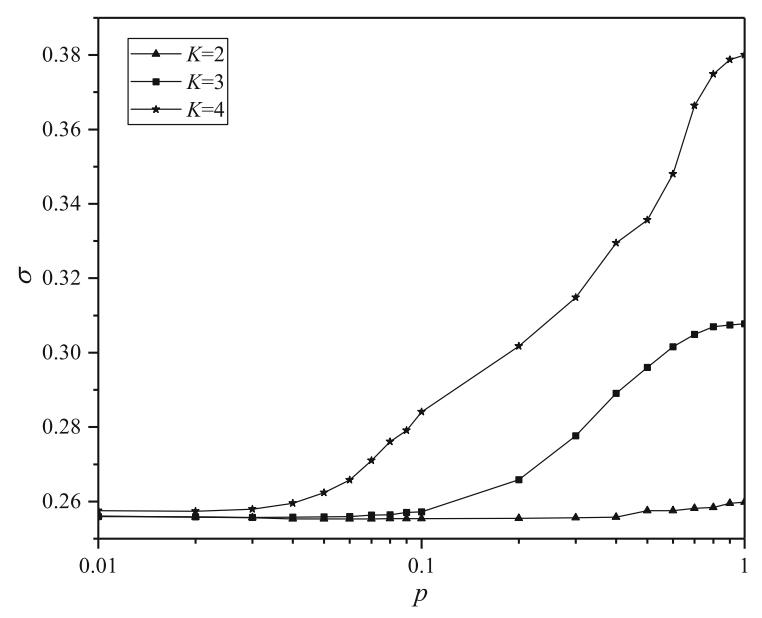

Fig. 7 Synchronization of the system as a function of the disorder parameter $p$ under coordination number $K$ with parameters $N=10^{4}, \tau_{I}=5, n_{\text {inf }}(0)=0.1, M_{t h}=4$

In Fig. 7, the synchronization parameter corresponding to coordination number $K=2,3$ and 4 are presented. Each point corresponds to a subsequent average over 20 realizations of the networks and the initial condition. The transition becomes sharper for large coordination number. With the increase of $\mathrm{K}$, the critical value $p_{\text {critical }}$ decreases. When $K$ increase from 2 to $4, p_{\text {critical }}$ decreases from 0.4 to 0.03 . This is reasonable, since higher $K$ means more interaction between individuals in the system, making the system approach to a globally coupled system. A highly globally coupled system is more prone to synchronous behavior. As 
Fig. 8 Synchronization of the system as a function of the disorder parameter $p$ under coordination number $K$ with parameters $N=10^{4}, K=12, \tau_{I}=$ $5, n_{\text {inf }}(0)=0.1, M_{t h}=4$

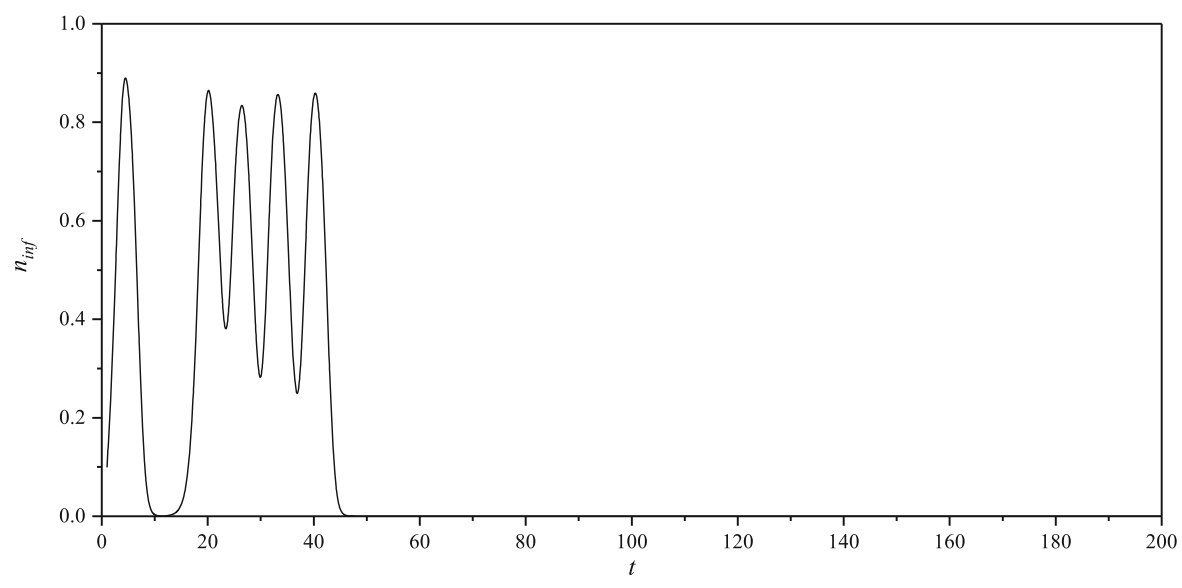

shown in Fig. 8, a further increase in $K$ will lead to a rapid spread of the epidemic throughout the network. At some points, the highly synchronized behavior leads to the status $\tau_{i}$ of all individuals is 0 at the same time, making the virus disappear in the system before mutation occurs.

In summary, the structure of the small-world network has an important impact on the spread and mutation of the epidemic. A highly globally coupled system is more prone to synchronous behavior. Thus, increasing the coordination number makes the system more prone to oscillations in the fraction of infected people, while simply increasing the size of the network does not have a significant effect on the transitions. This is because increase in $K$ enhances the individual interactions within the system and thus drives the system closer to the globally coupled system, while increasing the network size alone does not promote the globally coupled behavior of the system. For systems that tend to be more globally coupled, the virus spreads rapidly throughout the network, making the effective mutation parameter lower. While increasing the system size does not increase the global coupling behavior of the system, it does increase the absolute number of infected viruses. This is what makes the virus have a higher probability of being preserved in the network during propagation, which is equivalent to disguisedly extending the maximum valid mutation cycle of the virus.

In addition, there is a correlation between present work and the study in Ref. [17]. In Ref. [17], it is assumed that the virus does not mutate, but the individual's immune cycle to the virus is limited, which is called first case. In present work, it is considered that individuals can produce permanent immunity on the basis of virus mutation, which is called the second case. Obviously, in the small world network, the infection characteristics of viruses under the above two cases are highly similar. Further, compared with the results in Ref. [12], although the virus can mutate, the individual immune cycle to the virus is limited. However, the results in Ref. [12] are very similar to those in this paper. That is to say, the influence of the above two conditions on the characteristics of virus transmission in small world network can be regarded as the same. In fact, the above analysis mainly involves two key factors, that is, whether the virus can mutate into a new virus, and whether the individual is permanently immune to the virus. The above two factors can be combined into four situations: (1) the first condition is that the virus does not mutate, and individuals can be permanently immune to the virus. The traditional SIR model is based on this assumption. The transmission characteristic of the virus is that the only virus infects the whole population and produces herd immunity. The virus cannot continue to spread and therefore disappears; (2) The second condition is that the virus does not mutate and the immune cycle of individuals is limited. The research in Ref. [17] is based on this hypothesis; (3) the third condition is that the virus can mutate, and the individual immune cycle is limited. The research in Ref. [12] is based on this hypothesis; (4) The fourth condition is the case of this paper, the virus can mutate, individuals can produce permanent immunity to the virus.

These situations can be regarded as different situations in which human and virus compete and develop in the process of evolution. The latter three situations are different from the results of SIR model, but they are very similar to each other. Compared with the sit- 
uation that the virus can not survive in SIR model, if the immune cycle of human to the virus is limited, it will also improve the probability of the virus to continue to spread in the population. However, if humans evolved permanent immunity to the virus, the virus could continue to spread through mutation. In summary, the mutation is equivalent to shortening the human immune period to the virus. For the case of permanent immunity, the virus mutation is equivalent to making the immune cycle of human to the virus from unlimited to limited.

\subsection{Influences of mutation cycle on epidemic transmission}

Figure 9 shows the time-varying curve of the fraction of infected to the total population at different mutation thresholds $M_{t h}$. When $M_{t h}$ is small, the fraction of infected randomly fluctuates around a fixed value, and the fluctuation is very weak. Figure $9 b$, c shows that the fluctuation of some intervals in the time-varying curve of the fraction of infected gradually increases with $M_{t h}$. Continuing to increase $M_{t h}$, the curve will show an oscillation with moderate amplitude, as shown in Fig. 9d, e. As shown in Fig. 9f, g, a further increase in $M_{t h}$ results in a coexistence of large and moderate amplitude fluctuations in the curve. As $M_{t h}$ continues to increase to a certain value, the virus mutation cycle becomes longer. This leaves all individuals free of new virus infections until herd immunity occurs. Therefore, the epidemic cannot continue to spread on the network. With other parameters unchanged, $M_{t h}$ mainly determines whether the virus can continue to spread in the network. If a certain $M_{t h}$ can make the virus continue to spread in the network, it is called the invalid mutation parameter. If the virus has not mutated before it disappears under a certain $M_{t h}$, it is called invalid mutation parameter.

Next, the influences of network structure on the maximum valid mutation parameter are discussed. Due to the randomness of virus mutation and transmission, it is not reasonable to determine whether the parameter is a valid mutation parameter by only one simulation. In this paper, different mutation parameters are selected and 100 simulations are performed for each mutation parameter. If a parameter fails to continue the virus propagation in these 100 simulations, the parameter is considered as an invalid mutation parameter. Oth- erwise, the parameter is considered as a valid mutation parameter. As shown in Figs. 10 and 11, as the system size and coordination number are determined, the maximum valid mutation parameter is larger when the small-world network is more regular. The maximum valid mutation parameter decreases sharply as $p$ increases. As $p$ is greater than 0.2 , the maximum valid mutation parameter decreases slowly with the increase of $p$. From Fig. 10, it can be seen that the maximum valid mutation parameter curve shifts upward as the system size increases. This means that the maximum valid mutation parameter increases with $N$. On the contrary, it can be seen from Fig. 11 that the maximum valid mutation parameter curve will move downward with the increase of coordination number. That is to say, with the increase of $K$, the maximum valid mutation parameter will decrease. In a word, the size, disorder parameter and coordination number of the small-world network have an important impact on the spread of the epidemic.

Furthermore, the influence of infection rate $q$ on the transmission characteristics of the virus with mutation is discussed.

For different $M_{t h}$, the time-average fraction of the infected in 200-time steps after the infection curve entering the stationary state as a function of the infection rate are shown in Fig. 12. Each point in Fig. 12 corresponds to a subsequent average over 100 realizations of the networks and the initial condition. During virus transmission, it is not simply that higher infection rates will lead to larger and more persistent infections. As the infection rate approaches 0 , the time-average fraction of the infected also approaches 0 . As the infection rate increases, the fraction of the infected increases and then decreases. After the time-average fraction increases to a certain level, the time-average fraction goes to 0 . Obviously, the size of the infected is largest only at a moderate infection rate. Once the infection rate exceeds a certain value, the virus spreads rapidly throughout the network so that the population reaches a state of herd immunity and the virus disappears. More, as the mutation period increases, the time-average fraction of the infected decreases. The infection rate corresponding to the maximum time-average fraction of the infected also decreases. 


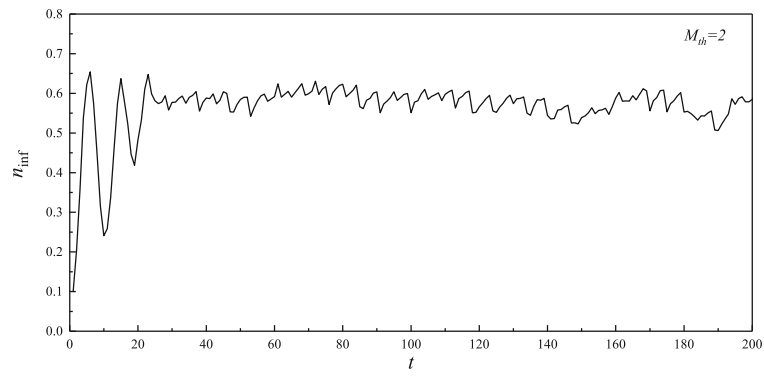

(a)

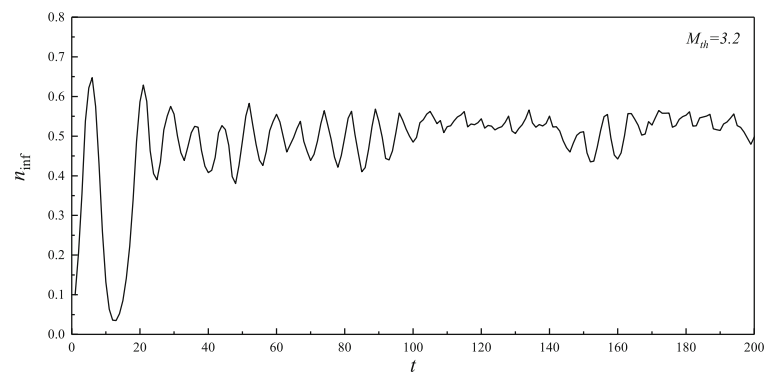

(c)

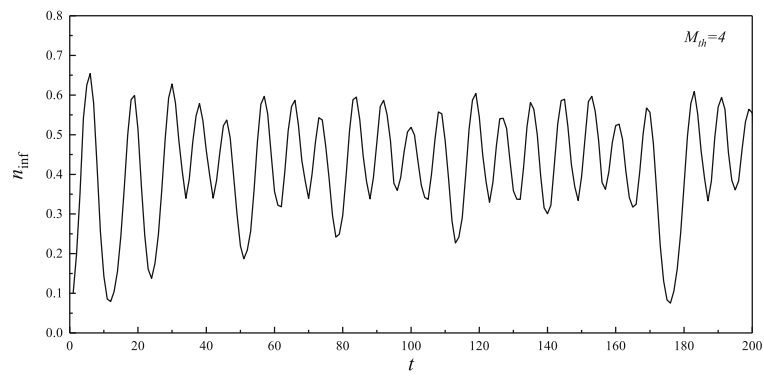

(e)

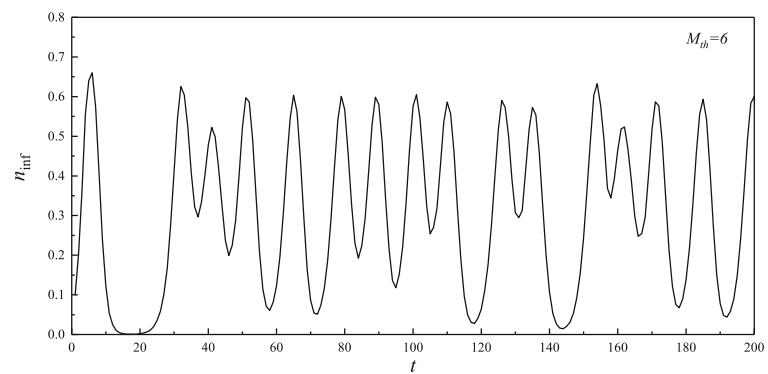

(g)

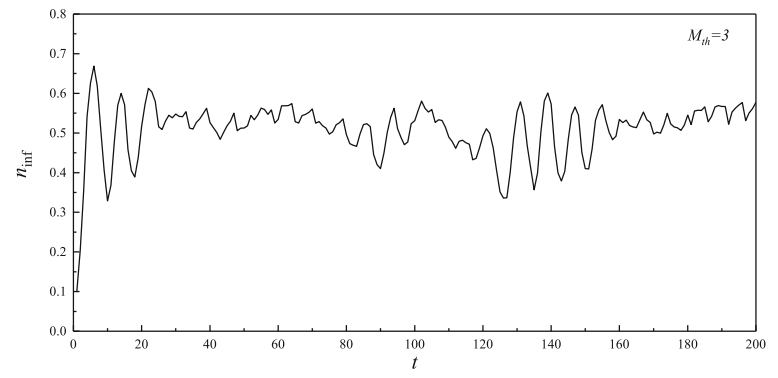

(b)

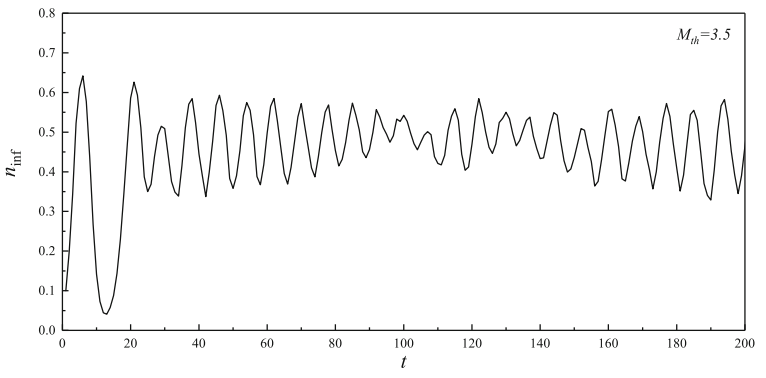

(d)

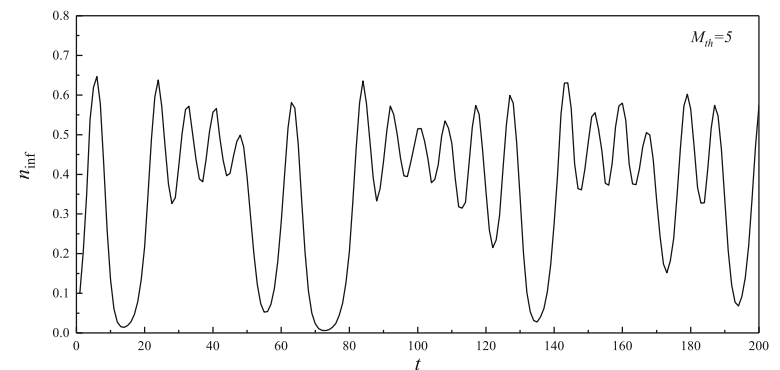

(f)

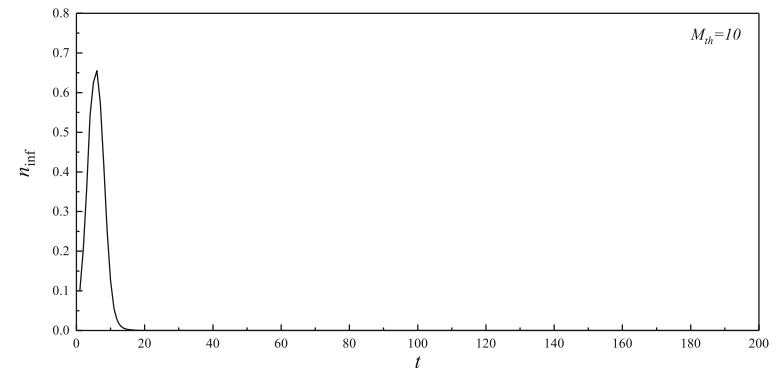

(h)

Fig. 9 Fraction of infected as a function of time under different mutation thresholds $M_{t h}$ with parameters $N=10^{4}, K=12, \tau_{I}=$ $5, n_{\text {inf }}(0)=0.1$ 


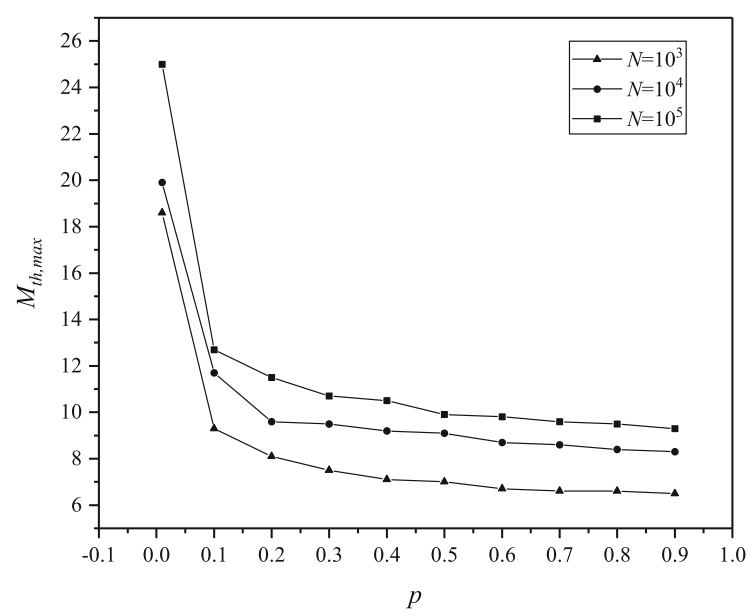

Fig. 10 Maximum valid mutation parameters as a function of disorder parameter $p$ under different system size $N$ with parameters $K=3, \tau_{I}=5, n_{\text {inf }}(0)=0.1$

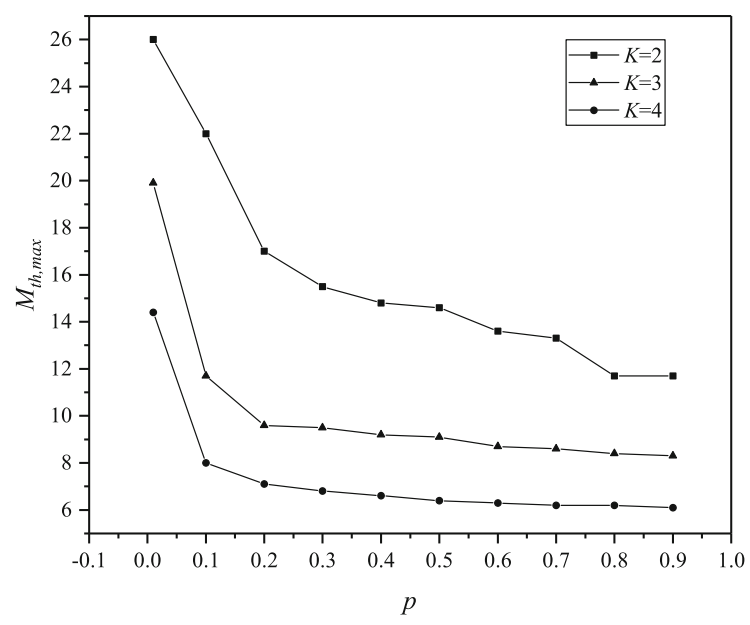

Fig. 11 Maximum valid mutation parameters as a function of disorder parameter $p$ under different coordination number $K$ with parameters $N=10^{4}, \tau_{I}=5, n_{\text {inf }}(0)=0.1$

\section{Conclusions}

In this paper, a novel virus mutation-immune model is proposed to study the network dynamics of the virus transmission that can mutate and be permanently immunized. The mutation-immune model combined with an modified SIR model to simulate the spread of epidemic in small-world network. First, the effects of the size, coordination number and disorder parameter of the small-world network on the spread of the epidemic are analyzed. Then, the effects of mutation cycle and infection rate on epidemic transmission in small-

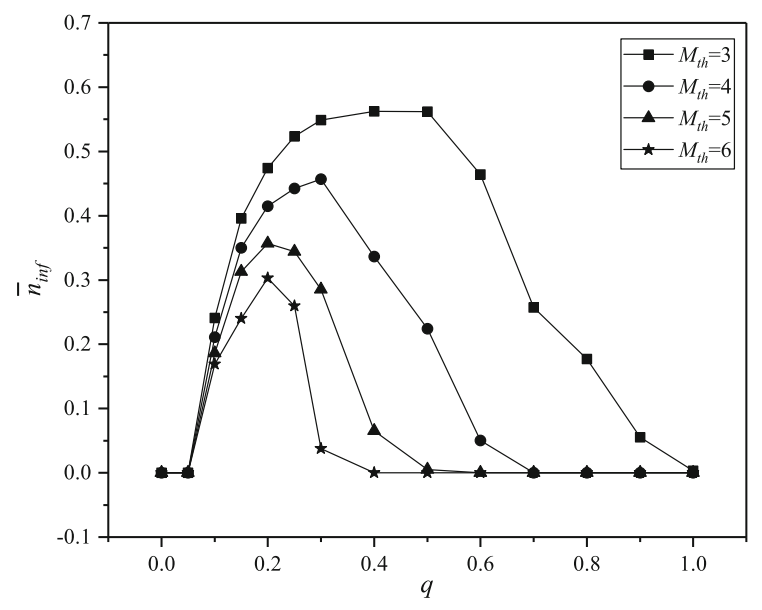

Fig. 12 Time-average fraction as a function of the infection rate under different $M_{t h}$ with parameters $N=10^{4}, K=3, p=$ $0.1, \tau_{I}=5, n_{\text {inf }}(0)=0.1$

world network are investigated. The main conclusions obtained are summarized as follows,

1. The structure of the small-world network has an important impact on the spread of the epidemic. When the disorder parameter changes from small to large, the transition can be observed on a certain disorder parameter. The dynamical behavior of the modified SIR epidemic model with mutationimmune mechanism changes from an irregular, low-amplitude evolution at small $p$ to a spontaneous state of wide amplitude oscillations at large $p$. Also, it can be seen that the critical disorder value $p_{\text {critical }}$ of transition is basically unchanged in different sizes. mechanism the increase of $K$, $p_{\text {critical }}$ shifts to a smaller value. After the transition, the synchronization parameters increase with the increase of $K$.

2. For the transmission of mutable virus in small world network, whether the immune cycle is finite or infinite, the transmission characteristics of virus are highly similar. This is because the essence of virus mutation is equivalent to shortening the period of human immune to virus. For the case of permanent immunity, the virus mutation is equivalent to making the immune cycle of human to the virus from infinite to finite.

3. Epidemic mutation cycle also has an important impact on the spread of the epidemic. When the mutation parameter is small, the fraction of infected fluctuates around a fixed value, and the transition 
occurs with the further increase of mutation parameters. However, when the mutation parameter is greater than to a certain value, the virus could not continue to spread. Moreover, the maximum valid mutation parameters decrease with the increase of disorder parameter and coordination number, but increase with the system size.

4. The effect of infection rate on virus spread is not simply that higher infection rates lead to larger and more persistent infections. As the infection rate increases, the fraction of the infected increases and then decreases. After the time-average fraction increases to a certain level, the epidemic disappears. More, as the mutation cycle increases, the timeaverage fraction of the infected and the infection rate corresponding to the maximum time-average fraction of the infected also decreases.

This paper investigates the transmission of mutable viruses that can be permanently immunized in smallworld networks and yields some important conclusions that can improve the understanding of the propagation properties of mutated viruses. However, there is still no further study the transition to synchronization occurs. This may be related to the existence of some specific structures in small world networks, which needs further study.

Acknowledgements This work is supported by Postdoctoral Research Foundation of China under Grant Nos. 2018M631140, and Youth program of National Natural Science Foundation of China under Grant Nos. 12002252.

Data availability The datasets generated during and/or analysed during the current study are available in the DataForND repository, https://github.com/csl199303/DataForND.

\section{Declarations}

Conflict of interest The authors declare that they have no conflict of interest.

\section{References}

1. Huang, C., Wang, Y., Li, X., Lili, R., Zhao, J., Hu, Y., Zhang, L., Fan, G., Xu, J., Gu, X., Cheng, Z., Yu, T., Xia, J., Wei, Y., Wu, W., Xie, X., Yin, W., Li, H., Liu, M., Xiao, Y., Gao, H., Guo, L., Xie, J., Wang, G., Jiang, R., Gao, Z., Jin, Q., Wang, J., Cao, B.: Clinical features of patients infected with 2019 novel coronavirus in Wuhan, China. Lancet 395(10223), 497-506 (2020)
2. Bontempi, E.: The Europe second wave of COVID-19 infection and the Italy "strange" situation. Environ. Res. (2020). https://doi.org/10.1016/j.envres.2020.110476

3. Seligmann, H., Iggui, S., Rachdi, M., Vuillerme, N., Demongeot, J.: Inverted covariate effects for first versus mutated second wave Covid-19: high temperature spread biased for young. Biology 9(8), 226 (2020)

4. Cacciapaglia, G., Cot, C., Sannino, F.: Second wave COVID19 pandemics in Europe: a temporal playbook. Sci. Rep. (2020). https://doi.org/10.1038/s41598-020-72611-5

5. Weissman, D., Alameh, M., Silva, T., Collini, P., Hornsby, H., Brown, R., LaBranche, C.C., Edwards, R.J., Sutherland, L., Sampa, Santra, Mansouri, K., Gobeil, S., McDanal, C., Pardi, N., Hengartner, N., Lin, P.J.C., Tam, Y., Shaw, P.A., Montefiori, D.C.: D614G spike mutation increases SARS CoV-2 susceptibility to neutralization. Cell Host Microbe 29(1), 23-31 (2020)

6. Korber, B., Fischer, W.M., Gnanakaran, S., Yoon, H., Theiler, J., Abfalterer, W., Hengartner, N., Giorgi, E.E., Bhattacharya, T., Foley, B., Hastie, K.M., Parker, M.D., Partridge, D.G., Evans, C.M., Freeman, T.M., Silva, T.I., Angyal, A., Brown, R.L., Carrilero, L., Green, L.R., Groves, D.C., Johnson, K.J., Keeley, A.J., Lindsey, B.B., Parsons, P.J., Raza, M., Rowland-Jones, S., Smith, N., Tucker, R.M., Wang, D., Wyles, M.D., McDanal, C., Perez, L.G., Tang, H., Moon-Walker, A., Whelan, S.P., LaBranche, C.C., Saphire, E.O., Montefiori, D.C.: Tracking Changes in SARS-CoV2 Spike: evidence that D614G increases infectivity of the COVID-19 Virus. Cell 182(4), 812-827.e19 (2020)

7. Koopmans, M.: SARS-CoV-2 and the human-animal interface: outbreaks on mink farms. Lancet Infect. Dis. 21(1), 18-19 (2021)

8. Davies, N.G., Abbott, S., Barnard, R.C., Jarvis, C.I., Kucharski, A.J., Munday, J.D., Pearson, C.A.B., Russell, T.W., Tully, D.C., Washburne, A.D., Wenseleers, T., Gimma, A., Waites, W., Wong, K.L.M., Zandvoort, K., Silverman, J.D., CMMID COVID-19 Working Group, The COVID19 Genomics UK (COG-UK) Consortium, Diaz-Ordaz, K., Keogh, R., Eggo, R.M., Funk, S., Jit, M., Atkins, K.E., Edmunds, W.J.: Estimated transmissibility and severity of novel SARS-CoV-2 Variant of Concern 202012/01 in England. MedRxiv (2021). https://doi.org/10.1101/2020.12.24. 20248822

9. Moore, C., Newman, M.E.J.: Epidemics and percolation in small-world networks. Phys. Rev. E. 61(5), 5678 (2020)

10. Sander, L.M., Warren, C.P., Sokolov, I.M., Koopman, J.: Percolation on heterogeneous networks as a model for epidemics. Math. Biosci. 180(1-2), 293-305 (2002)

11. Pastor-Satorras, R., Castellano, C., Van Mieghem, P., Vespignani, A.: Epidemic processes in complex networks. Rev. Mod. Phys. 87(3), 925 (2015)

12. Rüdiger, S., Plietzsch, A., Sagués, F., Sokolov, I.M., Jürgen, K.J.: Epidemics with mutating infectivity on smallworld networks. Sci. Rep. (2020). https://doi.org/10.1038/ s41598-020-72611-5

13. Watts, D.J., Strogatz, S.H.: Collective dynamics of 'smallworld' networks. Nature 393(6684), 440-442 (1998)

14. Watts, D.J.: The "new" science of networks. Annu. Rev. Sociol. 30, 243-270 (2004)

15. Barabási, A.L.: Linked: The New Science of Networks. Perseus, Cambridge (2002) 
16. Newman, M.E.J.: The structure and function of complex networks. SIAM Rev. 45(2), 167-256 (2003)

17. Kuperman, M., Abramson, G.: Small world effect in an epidemiological model. Phys. Rev. Lett. 86(13), 2909 (2001)

18. Small, M., Tse, C.K.: Clustering model for transmission of the SARS virus: application to epidemic control and risk assessment. Phys. A. 351(2-4), 499-511 (2005)

19. Wang, X.F., Chen, G.: Synchronization in small-world dynamical networks. Int. J. Bifurc. Chaos. 12(01), 187-192 (2002)

20. Li, K., Zhu, G., Ma, Z., Chen, L.: Dynamic stability of an SIQS epidemic network and its optimal control. Commun. Nonlinear Sci. Numer. Simul. 66, 84-95 (2019)

21. Boguná, M., Castellano, C., Pastor-Satorras, R.: Nature of the epidemic threshold for the susceptible-infectedsusceptible dynamics in networks. Phys. Rev. Lett. 111(6), 068701 (2013)

22. Liu, M., Xiao, Y.: Modeling and analysis of epidemic diffusion within small-world network. J. Appl. Math. 2012, 841531 (2012)

23. Santos, F.C., Rodrigues, J.F., Pacheco, J.M.: Epidemic spreading and cooperation dynamics on homogeneous small-world networks. Phys. Rev. E. 72(5), 056128 (2005)

24. Li, C., Jiang, G., Song, Y.: Comparative effects of avoidance and immunization on epidemic spreading in a dynamic small-world network with community structure. Wuhan Univ. J. Nat. Sci. 21(4), 291-297 (2016)

25. Saif, M.A.: Epidemic threshold for the SIRS model on the networks. Phys. A 535, 122251 (2019)

26. Xu, Z., Sui, D.Z.: Effect of small-world networks on epidemic propagation and intervention. Geogr. Anal. 41(3), 263-282 (2009)

27. Kabir, K.M.A., Tanimoto, J.: Analysis of epidemic outbreaks in two-layer networks with different structures for information spreading and disease diffusion. Commun. Nonlinear Sci. Numer. Simul. 72, 565-574 (2019)
28. Keeling, M.: The implications of network structure for epidemic dynamics. Theor. Popul. Biol. 67(1), 1-8 (2005)

29. Zhao, H., Gao, Z.Y.: Modular effects on epidemic dynamics in small-world networks. Europhys. Lett. 79(3), 38002 (2007)

30. Shao, Z.G., Tan, Z.J., Zou, X.W., Jin, Z.Z.: Epidemics with pathogen mutation on small-world networks. Phys. A 363(2), 561-566 (2006)

31. Girvan, M., Callaway, D.S., Newman, M.E.J., Strogatz, S.H.: Simple model of epidemics with pathogen mutation. Phys. Rev. E 65(3), 031915 (2002)

32. De Visser, J.A.G.M., Krug, J.: Empirical fitness landscapes and the predictability of evolution. Nat. Rev. Genet. 15(7), 480-490 (2014)

33. Rüdiger, S., Plietzsch, A., Sagués, F., Sokolov, I.M., Kurths, J.: Epidemics with mutating infectivity on small-world networks. Sci. Rep. 10(1), 5919 (2020)

34. Xu, D., Xu, X., Xie, Y., Yang, C.: Optimal control of an SIVRS epidemic spreading model with virus variation based on complex networks. Commun. Nonlinear Sci. Numer. Simul. 48(JUL), 200-210 (2017)

35. Xu, D., Xu, X., Yang, C., Gui, W.: Global stability of a variation epidemic spreading model on complex networks. Math. Probl. Eng. 2015, 1-8 (2015)

Publisher's Note Springer Nature remains neutral with regard to jurisdictional claims in published maps and institutional affiliations. 